\title{
Aa. Vv., Lexique, Syntaxe et Lexique-Grammaire - Syntax, Lexis \& Lexicon-Grammar. Papers in honour of Maurice Gross
}

\section{Sara Vecchiato}

\section{(2) OpenEdition \\ 1 Journals}

\section{Édition électronique}

URL : http://journals.openedition.org/studifrancesi/28383

DOI : $10.4000 /$ studifrancesi.28383

ISSN : 2421-5856

Éditeur

Rosenberg \& Sellier

\section{Édition imprimée}

Date de publication : 31 décembre 2006

Pagination : 658-659

ISSN : 0039-2944

\section{Référence électronique}

Sara Vecchiato, «Aa. VV., Lexique, Syntaxe et Lexique-Grammaire - Syntax, Lexis \& Lexicon-Grammar. Papers in honour of Maurice Gross », Studi Francesi [En ligne], 150 (L | III) | 2006, mis en ligne le 30 novembre 2015, consulté le 08 novembre 2020. URL : http://journals.openedition.org/studifrancesi/ 28383 ; DOI : https://doi.org/10.4000/studifrancesi.28383

Ce document a été généré automatiquement le 8 novembre 2020.

\section{cc) $(9)$}

Studi Francesi è distribuita con Licenza Creative Commons Attribuzione - Non commerciale - Non opere derivate 4.0 Internazionale. 


\section{Aa. Vv., Lexique, Syntaxe et Lexique- Grammaire - Syntax, Lexis \& Lexicon- Grammar. Papers in honour of Maurice Gross}

Sara Vecchiato

\section{RÉFÉRENCE}

Christian LeCLeRE, Eric LAPORTE, Mireille PIOT, Max SILBERzTEIN (ed.), Lexique, Syntaxe et Lexique-Grammaire - Syntax, Lexis \& Lexicon-Grammar. Papers in honour of Maurice Gross, Amsterdam / Philadelphia, John Benjamins Publishing Company, 2004, pp. 660.

1 Ces contributions, écrit Eric LAPORTE dans l'introduction (Foreword, pp. XI-XXI), entendaient être un présent à Maurice Gross dans une occasion joyeuse. Malheureusement il tomba malade entre temps, et ne put voir qu'une version préliminaire du volume avant de s'éteindre en 2001. C'est ainsi que ses collègues ont transformé ce recueil en un hommage à sa mémoire. L'introduction s'attache à illustrer la contribution pionnière que Maurice Gross a fournie en linguistique générale et française. Sa contribution couvre en effet aussi bien une dimension théorique, dans le développement de l'approche Lexique-grammaire, qu'une dimension d'application. Ensuite, Jean Claude CHEVALIER présente des passages d'un entretien avec Maurice Gross qui avait eu lieu en 1982. M. Gross parle de sa formation, de son expérience comme jeune chercheur, de la constitution du Laboratoire d'Automatique Documentaire et Linguistique. Quelques remarques épistémologiques de CHEVALIER complètent le cadre (Entretien avec Maurice Gross, pp. 1-10).

2 Le volume comprend une trentaine de contributions qui traitent de linguistique française et une vingtaine sur des langues différentes, européennes et extraeuropéennes, que nous ne pouvons que signaler ici. 
3 Antoinette BALIBAR-MRABTI présente une esquisse des problèmes posés par le traitement des adjectifs qui se situent dans le continuum forme libre - forme figée. Le procédé de listage des combinaisons possibles et leur analyse entre syntaxe, sémantique et rhétorique y sont discutés (Lexique-Grammaire et extensions lexicales, pp. 23-30). Hava BAT-ZEEV SHILDKROT décrit les étapes de l'établissement d'une concordance informatisée des verbes de l'ancien français. Elle présente le choix du corpus et la méthodologie de catalogation des variantes, des formes conjuguées et de possibilités distributionnelles (La constitution d'une concordance de verbes de l'ancien français, pp. 41-50). Andrée BoRILLO analyse les adjectifs qui se réfèrent aux parties du corps humain, trait caractérisant les textes de spécialité médicaux. Elle examine leur classification sémantique et catégorielle, leur distribution et leur rapport avec les substantifs dont ils sont dérivés, ou avec lesquels ils se trouvent en collocation (Les adjectifs dérivés de noms de parties $d u$ corps (Npc) dans les textes médicaux, pp. 51-62). Mirella CoNENNA illustre son élaboration de dictionnaires électroniques et d'automates pour l'analyse des proverbes. Le but de ces instruments est de reconnaître les proverbes, même défigés ou interrompus par des insertions, de les traduire, d'en répertorier les variantes et d'en marquer les césures intonatives (Principes d'analyse automatique des proverbes, pp. 91-103). Benoît DE CORNULIER étudie la valeur de la phrase incise, sorte de greffon métalinguistique à l'intérieur d'une phrase qui «mime» l'énoncé d'un autre sujet parlant. En particulier la postposition de l'incise et les changements de sa portée y sont analysés (Sur la valeur de l'«incise» et sa postposition, pp. 105-112). Blandine CouRTOIs compare les dictionnaires électroniques construits à l'intérieur du LADL pour l'anglais et le français. La structure globale des articles y est d'abord illustrée, pour passer ensuite à une description des niveaux de codification en ce qui concerne les informations lexicales, sémantiques et syntaxiques des lemmes (Dictionnaires électroniques DELAF anglais et français, pp. 113-124). Laurence DANLOS focalise son attention sur les relations temporelles à l'intérieur du cadre «Segmented Discourse Representation Theory». Elle étudie les cas de coréférence entre deux éventualités, notamment dans les discours généralisants et particularisants, pour lesquels sont proposées des relations de discours nouvelles (Coréférence événementielle entre deux phrases, pp. 137-154). Jean DUBoIs et Françoise DUBOIs-CHARLIER observent des constructions grammaticales où paraissent des pronoms relatifs, en argumentant qu'il ne s'agit pas de relativations enchainnant deux phrases, mais des remaniements d'une seule phrase à des fins de réorganisation thématique ou de mise en relief (Les relatifs de surface, pp. 175-184). André DUGAS consacre sa réflexion aux attributs du complément d'objet direct et indirect, reprenant ses études exhaustives sur 12000 verbes. Les emplois des attributs des compléments sont décrits, de même que les problèmes liés à cette description (Les attributs du complément d'objet, pp. 185-194). Cédrick FAIRON présente une analyse sur les phrases incises à partir d'un corpus littéraire et joumalistique. Le recensement d'environ 740 verbes lui permet de remettre en question l'affirmation traditionnelle que le verbe le plus fréquent en incise est dire et le temps le plus fréquent le participe passé (Une étude de corpus pour éclairer la question du verbe de l'incise en français, pp. 195-210). David GAATONE fait toucher du doigt la complexité d'un travail de définition catégorielle de la classe des prépositions. Leur nature hétérogène rend difficile en cerner un trait commun au niveau sémantique, lexical, ou syntaxique (Les prépositions forment-elles une classe?, pp. 211-222). L'étude de Jacqueline GIRYSCHNEIDER porte sur les expressions du type faire le fier. La division en deux typologies distinctes au niveau sémantique et syntaxique y est argumentée, contrairement à une 
tradition qui les traite comme des constructions à complément nominal homogènes et toutes également figées. (Une construction tronquée du verbe faire, pp. 223-230). Gaston Gross s'attache à démontrer l'importance de l'analyse par classes sémantiques dans la description linguistique, entre autres afin de cerner la sous-catégorisation des prédicats, décrire leur polysémie, en identifier les emplois dans un texte (Classes sémantiques et description des langues, pp. 231-238). Frantz GUENTHER et Xavier B lanco présentent une classification d'expressions figées et leur encodage dans les dictionnaires électroniques. Les composés, les collocations, les textes rituels y sont considérés, pour conclure avec des réflexions sur l'incidence de l'analyse grammaticale automatique en linguistique théorique (Multi-Lexemic Expressions: an overview, pp. 239-252). Richard KAYNE analyse les emplois locatifs et non locatifs des mots here et there en anglais, en appliquant une méthode comparative par rapport à la formulation de structures comparables en français et en italien (Here and There, pp. 253-274). Ferenc KIEFER discute l'ordre des adjectifs, en établissant une taxinomie fondée sur la complexité sémantique. Les adjectifs qui ont le comportement le plus irrégulier sont aussi les plus complexes (Sur l'ordre des adjectifs, pp. 275-286). Georges KLEIBER focalise l'intérêt sur l'anaphore associative. Par rapport à une certaine tradition qui l'identifie à l'anaphore indirecte, l'auteur prône une conception étroite qui restreint la notion d'anaphore associative à l'anaphore indirecte introduite par un article défini (Anaphores associatives: du large à l'étroit, pp. 287-302). Jacques LABELLE illustre le potentiel du cadre théorique du Lexique-grammaire pour la description de la microvariation à l'intérieur du français. Plusieurs différences sémantico-syntaxiques subtiles entre le français standard, le français de France et du Québec sont examinées (Lexiques-grammaires comparés. Quelques observations sur des différences syntaxiques en français de France et du Québec, pp. 313-324). Béatrice LAMIROY et Jean KLEIN travaillent sur un corpus de 500 verbes propres au français de Belgique. Les données montrent qu'à côté de la variation lexicale bien connue il existe une variation syntaxique: une taxinomie de 33 types verbaux est établie à l'intérieur du système linguistique belge ( $L a$ structure de la phrase en français de Belgique, pp. 343-372). Eric LAPORTE s'attache à formaliser en lexiquegrammaire la distribution des sujets des adjectifs - tâche qui est rendue difficile à cause de nombreuses restructurations et réductions métonymiques. Une taxinomie des constructions de départ est proposée (Restructuration and the subject of adjectives, pp. 373-388). Christian LECLERE et Jacqueline BRISBOIS-LEENHARDT présentent une approche de la synonymie axée sur la phrase. Contrairement à une analyse où l'on considère l'interchangeabilité des mots dans la même position, la mobilité des actants est à la base de cette conception (Synonymie de mots et synonymie de phrases: une approche formelle, pp. 389 404). Danielle LEEMAN étudie des structures du type La tarte, j'ai aimé, qui entrent apparemment dans la catégorie des topicalisation, pour montrer que des phénomènes d'accord, d'intonation, de syntaxe empèchent de parler, formellement, de topicalisation (Les aventures de Max et Eve, j'ai aimé. A propos d'un C.o.D. «Canada Dry», pp. 405-412). Claude MULLER travaille sur les structures de complémentation verbale introduites par ce que, les seules introduites par des prépositions. L'auteur analyse en particulier les propriétés des formes qui autorisent la complémentation complétive directe avec une interprétation indirecte (A propos de [pc-z], pp. 439 454). Kozué OGATA s'occupe de l'opposition entre le datif et le locatif directionnel, qui s'excluent mutuellement dans les constructions avec le verbe arriver. La priorité de la construction dative permet sa co-occurrence avec la locative si l'une est incluse dans 
l'autre (Du locatif directionnel au datif dans les constructions du verbe "arriver", pp. 471-484). Mireille PIOT conteste l'analyse traditionnelle de la séquence même si comme d'une seule conjonction concessive. Elle propose de la décomposer en si hypothéticoconditionnel, modifié par même, adverbe modifieur de conjonction (La conjonction “meme si" n'existe pas!, pp. 485-496). Antoinette Renouf mène une enquête sur la presence de gallicismes dans l'anglais contemporain. Elle parvient à démontrer que les fonctions et la perception des emprunts n'a guère changé depuis le xIII ${ }^{e}$ siècle (Shall we hors d'œuvres? The Assimilation of Gallicisms into English, pp. 527-546). Max SILBERZTEIN présente l'implémentation d'un analyseur syntaxique automatique de textes pour la description des déterminants et prédeterminants français. Des mots simples tels que le, mais aussi des séquences complexes sont formalisés à l'aide des graphes INTEX (Reconnaissance des déterminants français, pp. 589-600). Yoichiro TsuRUGA étudie un certain nombre de correspondances dans les structures du français, comme entre Luc charge le camion de blé et Luc charge du blé dans le camion; il propose une codification des facteurs réglant ces correspondances dans les tables du Lexique-grammaire (Essai d'interprétation fonctionnelle des tables du Lexique Grammaire, pp. 601-612). Robert VIVES illustre les résultats du travail du Laboratoire de Linguistique Informatique sur les prédicats nominaux, en expliquant les grilles d'encodage lexicales, syntaxiques et sémantiques utilisées (Une grille d'analyse pour les prédicats nominaux, pp. 641-648).

Le livre s'achève par une bibliographie complète des publications de Maurice Gross établie par Takuya NAKAMURA (Publications de Maurice Gross 1963-2002, pp. 649-659). 